\title{
THE $K$-THEORY OF ABELIAN SYMPLECTIC QUOTIENTS
}

\author{
Megumi Harada And Gregory D. Landweber
}

\begin{abstract}
Let $T$ be a compact torus and $(M, \omega)$ a Hamiltonian $T$-space. In a previous paper, the authors showed that the $T$-equivariant $K$-theory of the manifold $M$ surjects onto the ordinary integral $K$-theory of the symplectic quotient $M / / T$, under certain technical conditions on the moment map. In this paper, we use equivariant Morse theory to give a method for computing the $K$-theory of $M / / T$ by obtaining an explicit description of the kernel of the surjection $\kappa: K_{T}^{*}(M) \rightarrow K^{*}(M / / T)$. Our results are $K$-theoretic analogues of the work of Tolman and Weitsman for Borel equivariant cohomology. Further, we prove that under suitable technical conditions on the $T$-orbit stratification of $M$, there is an explicit Goresky-Kottwitz-MacPherson ("GKM") type combinatorial description of the $K$-theory of a Hamiltonian $T$-space in terms of fixed point data. Finally, we illustrate our methods by computing the ordinary $K$-theory of compact symplectic toric manifolds, which arise as symplectic quotients of an affine space $\mathbb{C}^{N}$ by a linear torus action.
\end{abstract}

\section{Introduction}

The main result of this manuscript is an explicit description of the ordinary $K$ theory of an abelian symplectic quotient $M / / T$ in terms of the equivariant $K$-theory $K_{T}^{*}(M)$ of the original Hamiltonian $T$-space $M$, where $T$ is a compact torus. Symplectic quotients by Lie groups arise naturally in many different fields; well-known examples are toric varieties and moduli spaces of bundles over Riemann surfaces. Further, symplectic quotients can often be identified with Geometric Invariant Theory ("GIT") quotients in complex algebraic geometry. Hence, many moduli spaces that arise as GIT quotients also have symplectic realizations, and the topological invariants of such moduli spaces give useful constraints on moduli problems. In addition, the theory of geometric quantization provides a fundamental link between the topology of symplectic quotients and representation theory (see e.g. [22, Section 7]). Our methods, as developed in this manuscript, give a general procedure for computing the $K$-theory of such spaces when the Lie group is a compact torus. By $K$-theory, we mean topological, integral $K$-theory, taking $K^{0}(X)$ to be the isomorphism classes of virtual complex vector bundles over $X$ when $X$ is compact, or for more general $X$, taking $[X, \operatorname{Fred}(\mathcal{H})]$ for a complex separable Hilbert space $\mathcal{H}$. In the equivariant case, by $K_{T}(X)$ we mean Atiyah-Segal $T$-equivariant $K$-theory [26], built from $T$-equivariant vector bundles if $X$ is a compact $T$-space, and $T$-equivariant maps $[X, \operatorname{Fred}(\mathcal{H})]_{T}$ if $X$ is a compact $T$-space, and $T$-equivariant maps $\left[X, \operatorname{Fred}\left(\mathcal{H}_{T}\right)\right]_{T}$ if $X$ is noncompact (here $\mathcal{H}_{T}$ contains every irreducible representation of $T$ with infinite multiplicity, see e.g. [4]).

Received by the editors January 4, 2007.

2000 Mathematics Subject Classification. Primary: 53D20; Secondary: $19 L 47$.

Key words and phrases. symplectic quotient, $K$-theory, equivariant $K$-theory, Kirwan map. 
In the setting of rational Borel equivariant cohomology, a fundamental symplectogeometric result of Kirwan [21] states that there is a natural surjective ring homomorphism

$$
\kappa_{H}: H_{T}^{*}(M ; \mathbb{Q}) \rightarrow H^{*}(M / / T ; \mathbb{Q}),
$$

where $H_{T}^{*}(M ; \mathbb{Q})$ is the $T$-equivariant cohomology ring of the original Hamiltonian $T$ space $M$ from which $M / / T$ is constructed. Hence, in order to compute $H^{*}(M / / T ; \mathbb{Q})$, it suffices to compute two objects: the equivariant cohomology $\operatorname{ring} H_{T}^{*}(M ; \mathbb{Q})$ and the kernel of $\kappa_{H}$. For both of these computations, one can use equivariant techniques that are unavailable on the quotient. Following Kirwan's original theorem [21], this "Kirwan method" has been well developed to yield explicit methods to compute both $H_{T}^{*}(M ; \mathbb{Q})$ and $\operatorname{ker}\left(\kappa_{H}\right)$. In this paper, we generalize to $K$-theory the explicit computation of the kernel of $\kappa_{H}$ given by Tolman and Weitsman [28]. Further, we also take to the $K$-theory setting the GKM-type combinatorial description of the $T$-equivariant cohomology of Hamiltonian $T$-spaces, which is motivated by the original work of Goresky, Kottwitz, and MacPherson [11].

We now explain the setting of our results. Suppose that $M$ is a symplectic manifold with a Hamiltonian $T$-action, i.e., there exists a moment map $\mu: M \rightarrow \mathfrak{t}^{*}$. Assuming that $T$ acts freely on the level set $\mu^{-1}(0)$, the symplectic quotient is then defined as $M / / T:=\mu^{-1}(0) / T$. We wish to compute the ordinary $K$-theory $K^{*}(M / / T)$. The first step in this direction is the $K$-theoretic analogue of the Kirwan surjectivity result (1.1) above, proven in [16]. Just as in the cohomology case, there is a natural ring homomorphism $\kappa$ induced by the natural inclusion $\mu^{-1}(0) \hookrightarrow M$ as follows:

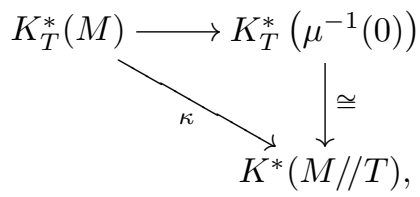

and $\kappa$ is surjective. The main result of this paper is to give an explicit computation of the kernel of the $K$-theoretic Kirwan map $\kappa$ above. This, together with the surjectivity of $\kappa$, gives us a method to explicitly describe the $K$-theory of abelian symplectic quotients.

A key element in our arguments is the $K$-theoretic Atiyah-Bott lemma, which is a $K$-theoretic analogue of a fact originally proven in [3] in the setting of Borel equivariant cohomology. In this manuscript, we use the formulation given in [16, Lemma 2.1]; a version in the algebraic category is given in [29, Lemma 4.2]. As we noted in [16], the Atiyah-Bott lemma is a crucial step in many Morse-theoretic proofs in symplectic geometry using the moment map $\mu$, and once we have a $K$-theoretic Atiyah-Bott lemma, it may be expected that many symplectic-geometric results in the setting of rational Borel equivariant cohomology carry over to that of (integral) $K$-theory. Indeed, the results of this manuscript can be viewed as illustrations of this principle.

We now briefly outline the contents of this manuscript. In Section 2 we prove an injectivity result, Theorem 2.5, which states that the $T$-equivariant $K$-theory of the Hamiltonian $T$-space, under some technical conditions on the moment map, injects into the $T$-equivariant $K$-theory of the fixed point set $M^{T}$ via the natural restriction 
$\operatorname{map} \imath^{*}: K_{T}^{*}(M) \rightarrow K_{T}^{*}\left(M^{T}\right)$. Similar results were obtained in the compact Hamiltonian setting already in [13], and in the algebraic category (in algebraic $K$-theory, for actions of diagonalizable group schemes on smooth proper schemes over perfect fields) in [29]. Our contribution is to extend the result in the Hamiltonian setting to a situation where $M$ may not be compact; the proof uses standard Morse-theoretic techniques in symplectic geometry using a generic component of the moment map and the $K$-theoretic Atiyah-Bott lemma mentioned above. This injectivity result is an important preliminary step for our main result because the $T$-action on $M^{T}$ is trivial, and the ring isomorphism $K_{T}^{*}\left(M^{T}\right) \cong K^{*}\left(M^{T}\right) \otimes K_{T}^{*}(\mathrm{pt})$ makes the equivariant $K$-theory of the fixed point set straightforward to compute.

With these tools in hand, we prove the following in Section 3:

Theorem 1.1. Let $T$ be a compact torus and $(M, \omega)$ a Hamiltonian $T$-space with moment map $\mu: M \rightarrow \mathfrak{t}^{*}$. Suppose there exists a component of the moment map which is proper and bounded below, and further suppose that $M^{T}$ has only finitely many connected components. Let

$$
Z:=\left\{\mu(C) \mid C \text { a connected component of } \operatorname{Crit}\left(\|\mu\|^{2}\right) \subseteq M\right\} \subseteq \mathfrak{t}^{*} \cong \mathfrak{t}
$$

be the set of images under $\mu$ of connected components of the critical set of the normsquare of $\mu$. Suppose that $T$ acts freely on $\mu^{-1}(0)$ and let $M / / T:=\mu^{-1}(0) / T$ be the symplectic quotient. For $\xi \in \mathfrak{t}$, define

$$
\begin{aligned}
M_{\xi} & :=\{x \in M \mid\langle\mu(x), \xi\rangle \leq 0\}, \\
\mathcal{K}_{\xi} & :=\left\{\alpha \in K_{T}^{*}(M)|\alpha|_{M_{\xi}}=0\right\}, \quad \text { and } \\
\mathcal{K} & :=\sum_{\xi \in Z \subseteq \mathfrak{t}} \mathcal{K}_{\xi} .
\end{aligned}
$$

Then there is a short exact sequence

$$
0 \longrightarrow \mathcal{K} \longrightarrow K_{T}^{*}(M) \stackrel{\kappa}{\longrightarrow} K^{*}(M / / T) \longrightarrow 0,
$$

where $\kappa: K_{T}^{*}(M) \rightarrow K^{*}(M / / T)$ is the $K$-theoretic Kirwan map.

This theorem is a $K$-theoretic analogue and a slight refinement of the computation of the kernel of the Kirwan map for rational Borel equivariant cohomology given by Tolman and Weitsman [28]. In their paper, Tolman and Weitsman give an expression for the kernel $\operatorname{ker}\left(\kappa_{H}\right)$ which is a sum of ideals $\mathcal{K}_{\xi}$ (defined similarly to those given above) over all $\xi \in \mathfrak{t}$, and it is not immediately evident that such an infinite sum, in the case when $M$ is not compact, yields a finite algorithm for the computation of the kernel. In our version of the computation, we refine the statement by explicitly exhibiting $\mathcal{K}$ as a sum over a finite set $Z$. For a discussion of a different simplification of the kernel computation for rational Borel equivariant cohomology in the compact Hamiltonian case, see [10].

In Section 4 we prove a $K$-theoretic version of a Goresky-Kottwitz-MacPherson ("GKM") combinatorial description of the $T$-equivariant $K$-theory of a Hamiltonian $T$-space satisfying certain conditions on the orbit type stratification. In this setting, we give an explicit combinatorial description of the image of $\imath^{*}$ in $K_{T}^{*}\left(M^{T}\right) \cong$ $K^{*}\left(M^{T}\right) \otimes K_{T}^{*}(\mathrm{pt})$. This result should be interpreted as a part of a large body of work motivated by, and in many ways generalizing, the original work of Goresky, Kottwitz, 
and MacPherson [11], who considered algebraic torus actions on projective algebraic varieties and Borel equivariant cohomology with $\mathbb{C}$ coefficients. For instance, it is now known that similar GKM results hold in the setting of Borel equivariant cohomology for Hamiltonian $T$-spaces (see e.g. [27, 15]). Similar GKM-type results for Hamiltonian $T$-spaces in equivariant $K$-theory with $\mathbb{C}$ coefficients are discussed in $[23,13]$, for other equivariant cohomology theories (under suitable hypotheses on the cohomology theory) and for more general $T$-spaces in [14], and for equivariant $K$-theory in the algebraic category in [29]. Our contribution in this section is to prove, using results in [14], that such a GKM theorem also holds in equivariant $K$-theory (over $\mathbb{Z}$ ) for Hamiltonian $T$-spaces which satisfy certain conditions on the $T$-orbit stratification.

Finally, in Section 5 we use our methods to give a computation of the ordinary $K$-theory of smooth compact projective toric varieties, which can be obtained by a symplectic quotient of an affine space $\mathbb{C}^{N}$. This rederives, using symplectic Morsetheoretic techniques, a description of the $K$-theory of these toric varieties analogous to the Stanley-Reisner presentation (see e.g. [29, Section 6.2], [5]).

The work in this manuscript opens many avenues for future research, of which we now mention a few examples. First, it would be of interest to give explicit computations of the $K$-theory of more examples of abelian symplectic quotients, such as polygon spaces $[18,19]$ or, more generally, weight varieties [9]. Furthermore, although we restrict our attention in this manuscript to the case where the symplectic quotient $M / / G$ is a manifold, we expect that an orbifold version of our results will still hold in the situation where $G$ acts only locally freely on $\mu^{-1}(0)$, making the quotient $\mathcal{X}=\mu^{-1}(0) / G$ an orbifold. Here we use the definition of the "full orbifold $K$-theory" $\mathrm{K}_{\mathrm{orb}}(\cdot)$ of an orbifold given in [20], where it is also shown that there is an orbifold Chern character map from the full orbifold $K$-theory $\mathrm{K}_{\text {orb }}(\mathcal{X})$ to the Chen-Ruan orbifold cohomology $H_{C R}^{*}(\mathcal{X})$ of the orbifold $\mathcal{X}$. Methods for computing the Chen-Ruan orbifold cohomology of orbifold symplectic quotients were given in [8] by using Kirwan surjectivity methods in addition to explicit computations of the kernel of the Kirwan map in the manifold case. We expect that, using the results of this manuscript and an approach similar to [8], we can also compute the full orbifold $K$-theory of orbifold symplectic quotients. Finally, it would be of interest to prove a $K$-theoretic analogue of the simplification of the computation of $\operatorname{ker}(\kappa)$ given by Goldin in [10] in the case of rational Borel equivariant cohomology. Goldin restricted her considerations to the case of compact Hamiltonian spaces in [10], but we expect that a similar statement should still hold. We intend to explore these and related topics in future work.

\section{Injectivity into the $K$-theory of the fixed points}

We begin with a $K$-theoretic version of the injectivity theorem of Kirwan, which is a key technical tool that we will need in the later sections. Suppose given $M$ a Hamiltonian $T$-space with moment map $\mu: M \rightarrow \mathfrak{t}^{*}$, with a component which is proper and bounded below. In this section only, we will for notational convenience use $\Sigma$ to denote the $T$-fixed point set $M^{T}$ of $M$. We additionally assume that $\Sigma$ has only finitely many connected components. In this situation, it is well-known that the inclusion $\imath: \Sigma \hookrightarrow M$ induces a map in rational Borel equivariant cohomology

$$
\imath^{*}: H_{T}^{*}(M ; \mathbb{Q}) \longrightarrow H_{T}^{*}(\Sigma ; \mathbb{Q}),
$$


which is an injection. We will prove a $K$-theoretic version of this injectivity (2.2) by Morse theory using a generic component of the $T$-moment map $\mu$. Our proof will follow that given in [15, Theorem 2.6].

Recall that the components $\mu^{\xi}:=\langle\mu, \xi\rangle$ for $\xi \in \mathfrak{t}$ of the moment map are MorseBott functions on $M$. We call a component generic if the critical set of $\mu^{\xi}$ is precisely the fixed point set $\Sigma$. This is true of an open dense set of directions $\xi \in \mathfrak{t}$. Our assumption above that there exists a component which is proper and bounded below guarantees that our Morse-theoretic arguments will work.

In the course of the proof we will use a special case of the $K$-theoretic Atiyah-Bott lemma, which we restate here for reference.

Lemma 2.1. ([29, Lemma 4.2], [16, Lemma 2.3]) Let a compact connected Lie group $G$ act fiberwise linearly on a complex vector bundle $\pi: E \rightarrow X$ over a compact connected $G$-manifold $X$. Assume that a circle subgroup $S^{1} \subseteq G$ acts on $E$ so that the fixed point set is precisely the zero section $X$. Choose an invariant metric on $E$ and let $D(E)$ and $S(E)$ denote the disc and sphere bundles, respectively. Then the long exact sequence for the pair $(D(E), S(E))$ in equivariant $K$-theory splits into short exact sequences

$$
0 \longrightarrow K_{G}^{*}(D(E), S(E)) \longrightarrow K_{G}^{*}(D(E)) \longrightarrow K_{G}^{*}(S(E)) \longrightarrow 0 .
$$

Remark 2.2. Recall that the splitting of the long exact sequence is equivalent, by the Thom isomorphism, to the statement that the $K$-theoretic equivariant Euler class of $E$ is not a zero divisor in $K_{G}^{*}(X)$. We will mainly use this latter perspective.

In what follows, we need only consider the Atiyah-Bott lemma for the case $G=T$. Moreover, in our applications of Lemma 2.1, the $T$-action on the base manifold $X$ will be trivial (since the base manifold is a component of a $T$-fixed set). Hence, in order to use the lemma, we need only check that there exists a subgroup $S^{1} \subseteq T$ acting on $E$ fixing precisely the zero section.

Before proceeding to the main argument, we prove the following simple technical lemma:

Lemma 2.3. Let $T$ be a compact torus and $(M, \omega)$ a Hamiltonian $T$-space with moment map $\mu: M \rightarrow \mathfrak{t}^{*}$. Suppose there exists a component of $\mu$ which is proper and bounded below, and further suppose that $\Sigma$ has only finitely many connected components. Then there exists a generic component $f:=\mu^{\xi}$ of $\mu$ which is proper, bounded below, and with respect to which all components of $\Sigma$ have different critical values.

Proof. If there is a component $\mu^{\eta}$ of $\mu$ which is proper and bounded below, then $\mu$ itself is proper, since a component is given by a linear projection. A result of Lerman, Meinrenken, Tolman, and Woodward [25, Theorem 4.2] states that in this situation the image $\mu(M)$ of $M$ is convex and locally polyhedral. Thus, by taking a small enough perturbation $\xi$ of $\eta$, we may arrange that all components of the fixed point set $M^{T}$ have different critical values with respect to $\mu^{\xi}$ and still have $\mu^{\xi}$ proper and bounded below.

With this lemma in hand, we may now state the proposition which is our main technical tool to show injectivity. This is the $K$-theoretic analogue of [15, Proposition 2.4]. 
Proposition 2.4. Let $T$ be a compact torus and $(M, \omega)$ a Hamiltonian $T$-space with moment map $\mu: M \rightarrow \mathfrak{t}^{*}$. Suppose there exists a component of the moment map which is proper and bounded below, and further suppose that $\Sigma$ has only finitely many connected components. Let $f:=\mu^{\xi}$ be a generic component of $\mu$ as in Lemma 2.3. Let $c$ be a critical value of $f$, and pick $\varepsilon>0$ such that $c$ is the only critical value of $f$ in $(c-\varepsilon, c+\varepsilon)$. Let $\Sigma_{c}$ be the component of $\Sigma$ with $f\left(\Sigma_{c}\right)=c$, and let $M_{c}^{ \pm}:=$ $f^{-1}(-\infty, c \pm \varepsilon)$. Then the long exact sequence of the pair $\left(M_{c}^{+}, M_{c}^{-}\right)$splits into short exact sequences

$$
0 \longrightarrow K_{T}^{*}\left(M_{c}^{+}, M_{c}^{-}\right) \longrightarrow K_{T}^{*}\left(M_{c}^{+}\right) \stackrel{k^{*}}{\longrightarrow} K_{T}^{*}\left(M_{c}^{-}\right) \longrightarrow 0,
$$

where $k: M_{c}^{-} \hookrightarrow M_{c}^{+}$is the inclusion. Moreover, the restriction map $K_{T}^{*}\left(M_{c}^{+}\right) \rightarrow$ $K_{T}^{*}\left(\Sigma_{c}\right)$, given by the inclusion $\Sigma_{c} \hookrightarrow M_{c}^{+}$, induces an isomorphism from the kernel of $k^{*}$ to the classes of $K_{T}^{*}\left(\Sigma_{c}\right)$ that are multiples of the equivariant Euler class e $\left(\Sigma_{c}\right)$ of the negative normal bundle to $\Sigma_{c}$ with respect to $f$.

Proof. This is a $K$-theoretic version of the proof given in [27]. Let $D\left(\nu_{c}\right)$ and $S\left(\nu_{c}\right)$ denote the disc and sphere bundles of the negative normal bundle $\nu_{c}$ to the fixed set $\Sigma_{c}$ with respect to $f$. By the assumption on $\varepsilon$, the component $\Sigma_{c}$ is the only component of $\operatorname{Crit}(f)$ in $f^{-1}(c-\varepsilon, c+\varepsilon)$. Similarly since $f$ is proper and $T$-invariant, the negative gradient flow with respect to $f$ using a $T$-invariant metric gives a $T$ equivariant retraction of the pair $\left(M_{c}^{+}, M_{c}^{-}\right)$to the pair $\left(D\left(\nu_{c}\right), S\left(\nu_{c}\right)\right)$. Using the $T$-equivariant Thom isomorphism, we conclude that the long exact sequence of the pair

$$
\cdots \rightarrow K_{T}^{*}\left(M_{c}^{+}, M_{c}^{-}\right) \rightarrow K_{T}^{*}\left(M_{c}^{+}\right) \rightarrow K_{T}^{*}\left(M_{c}^{-}\right) \rightarrow \cdots
$$

splits into short exact sequences if and only if the equivariant Euler class $e\left(\nu_{c}\right)$ is not a zero divisor. (We also note that, unlike the rational cohomology case, since $f$ is a component of a moment map, the Morse index of $f$ is even. Since $K_{T}^{*-\lambda}\left(D\left(\nu_{c}\right)\right) \cong$ $K_{T}^{*}\left(D\left(\nu_{c}\right)\right)$ by Bott periodicity, there is no degree shift here.) Moreover, the negative normal bundle $E$ is complex by a result of Kirwan [21]. The group $T$ fixes exactly the zero section $\Sigma_{c}$ because $\Sigma_{c}$ is defined to be a component of the $T$-fixed point set. We may apply Lemma 2.1 taking $G=T, X=\Sigma_{c}$, and $E=\nu_{c}$, together with a suitable choice of $S^{1} \subset T$, to conclude that $e\left(\Sigma_{c}\right)$ is not a zero divisor. The proposition follows.

We now come to the main theorem of this section. With Lemmas 2.1 and 2.3 and Proposition 2.4 in hand, the proof now exactly follows that given for [15, Theorem $2.5]$, so we will not reproduce it here.

Theorem 2.5. Let $T$ be a compact torus and $(M, \omega)$ a Hamiltonian $T$-space with moment map $\mu: M \rightarrow \mathfrak{t}^{*}$. Suppose there exists a component of the moment map which is proper and bounded below, and further suppose that $\Sigma$ has only finitely many connected components. Let $f:=\mu^{\xi}$ be a generic component of $\mu$ as in Lemma 2.3. Let $\imath: \Sigma \hookrightarrow M$ be the inclusion of the fixed point set into $M$. Then the restriction map

$$
\imath^{*}: K_{T}^{*}(M) \rightarrow K_{T}^{*}(\Sigma)
$$

is injective. 
Remark 2.6. Since the Atiyah-Bott lemma and Proposition 2.4 tell us that the Euler classes $e\left(\Sigma_{c}\right)$ of the negative normal bundles to the fixed point components are not zero divisors, in order to obtain the injectivity (2.2), we could also take the Morse stratification $M=\bigsqcup_{c} S_{c}$, where the $S_{c}$ are the flow-down manifolds associated to $\Sigma_{c}$, and apply the injectivity theorem [14, Theorem 2.3], which holds for more general equivariant cohomology theories. We present this straightforward Morse-theoretic argument here since we expect it to be more familiar to some readers.

\section{The kernel of the Kirwan map}

We have already observed in the introduction that in order to make explicit computations of the $K$-theory rings of abelian symplectic quotients, it is necessary to explicitly identify the kernel of the Kirwan map $\kappa: K_{T}^{*}(M) \rightarrow K^{*}(M / / T)$. In the setting of rational Borel equivariant cohomology, Tolman and Weitsman showed in [28] that the kernel of $\kappa$ can be identified as a sum of certain ideals in $H_{T}^{*}(M ; \mathbb{Q})$. In this section we state and prove the (integral) $K$-theoretic analogue of this result. This will allow us to make the explicit computation in Section 5.

Before proceeding to the main theorem, we take a moment to discuss the relationships between the various technical Morse-theoretic hypotheses on a moment map $\mu$ which are used in the literature. These are:

(1) there exists a component $\mu^{\xi}$ of the moment map which is proper and bounded below,

(2) $\mu$ is proper, and

(3) $\|\mu\|^{2}$ is proper.

It is straightforward to see that (1) implies (2), and that (2) and (3) are equivalent. When the Hamiltonian space $M$ is compact, then any of these conditions automatically holds; the point is that when $M$ is not compact, these conditions ensure that the Morse-theoretic arguments given below using $\mu$ still work. For the proof of the $K$-theoretic Kirwan surjectivity theorem in [16], we need only the second (or equivalently third) condition, namely, that $\mu$ is proper. However, for our explicit kernel computations we will be using Theorem 2.5 which requires the hypothesis (1) and additionally the condition that $M^{T}$ has finitely many components, so we continue making these stronger assumptions. In practice this is not very restrictive. ${ }^{1}$ Moreover, our hypotheses guarantee that the algorithm we present for computing the kernel of $\kappa$ is finite, as will be seen in Lemma 3.2.

We first give the statement of the main theorem of this section. In the following and in the sequel, we identify $\mathfrak{t} \cong \mathfrak{t}^{*}$ using an inner product on $\mathfrak{t}$, so the moment map $\mu$ can be considered as a map taking values in $t$.

Theorem 3.1. Let $T$ be a compact torus and $(M, \omega)$ a Hamiltonian $T$-space with moment map $\mu: M \rightarrow \mathfrak{t}^{*}$. Suppose there exists a component of the moment map which is proper and bounded below, and further suppose that $M^{T}$ has only finitely

\footnotetext{
${ }^{1}$ For instance, although hyperkähler quotients are rarely compact, they often admit natural Hamiltonian torus actions which do satisfy (1); for example, for hypertoric varieties, this fact was exploited in [15] to give combinatorial descriptions of their equivariant cohomology rings, and for hyperpolygon spaces, Konno uses a Hamiltonian $S^{1}$-action satisfying (1) in his proof of Kirwan surjectivity for these spaces [24]. (See also [17] for more on examples of this type.)
} 
many connected components. Let

$$
Z:=\left\{\mu(C) \mid C \text { a connected component of } \operatorname{Crit}\left(\|\mu\|^{2}\right) \subseteq M\right\} \subseteq \mathfrak{t}^{*} \cong \mathfrak{t}
$$

be the set of images under $\mu$ of components of the critical set of $\|\mu\|^{2}$. Suppose that $T$ acts freely on the level set $\mu^{-1}(0)$, and let $M / / T$ be the symplectic quotient. For $\xi \in \mathfrak{t}$, define

$$
\begin{aligned}
M_{\xi} & :=\{x \in M \mid\langle\mu(x), \xi\rangle \leq 0\}, \\
\mathcal{K}_{\xi} & :=\left\{\alpha \in K_{T}^{*}(M)|\alpha|_{M_{\xi}}=0\right\}, \quad \text { and } \\
\mathcal{K} & :=\sum_{\xi \in Z} \mathcal{K}_{\xi} .
\end{aligned}
$$

Then there is a short exact sequence

$$
0 \longrightarrow \mathcal{K} \longrightarrow K_{T}^{*}(M) \stackrel{\kappa}{\longrightarrow} K^{*}(M / / T) \longrightarrow 0
$$

where $\kappa: K_{T}^{*}(M) \rightarrow K^{*}(M / / T)$ is the Kirwan map.

We note that Theorem 3.1 is both a $K$-theoretic analogue of the kernel computation in $H_{T}^{*}(-; \mathbb{Q})$ by Tolman and Weitsman $[28$, Theorem 4] and a slight refinement of their statement, as we now explain. In [28, Theorem 4], the kernel is expressed as a sum over the infinite set of all elements in $\mathfrak{t}$. In the case where $M$ is compact, they comment that their algorithm is in fact finitely computable in [28, Remark 5.3], but they do not explicitly address what happens in the non-compact situation. In Theorem 3.1 we have expressed $\mathcal{K}$ as a sum over a certain set $Z$, which is shown to be finite in Lemma 3.2 below. The point is that our $K$-theoretic kernel $\mathcal{K}$ is explicitly computed by a finite algorithm even in the non-compact case.

We now show that $Z$ is finite. Recall that under our properness hypotheses, both $\mu$ and $\|\mu\|^{2}$ are proper.

Lemma 3.2. Let $T$ be a compact torus and $(M, \omega)$ a Hamiltonian $T$-space with moment map $\mu: M \rightarrow \mathfrak{t}^{*}$. Suppose there exists a component of the moment map which is proper and bounded below, and further suppose that $M^{T}$ has only finitely many connected components. Then there are only finitely many connected components of the critical set of the norm-square $\|\mu\|^{2}: M \rightarrow \mathbb{R}_{\geq 0}$ of the moment map. In particular, the set $Z$ in (3.1) is finite.

Proof. For a subgroup $H \subseteq T$ of the torus $T$, let $M_{H}$ denote the subset of $M$ consisting of points $p$ with $\operatorname{Stab}(p)=H$. Let

$$
M=\bigsqcup_{H \subseteq T} M_{H}
$$

be the orbit type stratification of $M$ as in [12, Section 3.5]. Since $T$ is compact, the action is proper. By standard Hamiltonian geometry [6] and the theory of proper group actions (see e.g. [12, Appendix B]), the closure $\overline{M_{H}^{(i)}}$ of each connected component $M_{H}^{(i)}$ of $M_{H}$ is itself a closed Hamiltonian $T$-space with moment map given by restriction. Since the restriction of $\Phi$ to $\overline{M_{H}^{(i)}}$ is also proper and bounded below, each $\overline{M_{H}^{(i)}}$ contains a $T$-fixed point. By equivariant Darboux, any $T$-invariant tubular neighborhood of a connected component of $M^{T}$ intersects only finitely many $T$-orbit types. 
Since by assumption there are only finitely many connected components of $M^{T}$, we may conclude that there are only finitely many orbit types in the decomposition (3.2), and that each $M_{H}$ has only finitely many connected components. Kirwan proves [21, Lemma 3.12] that there is at most one critical value of $\|\mu\|^{2}$ on each $\Phi\left(\left(M_{H}\right)^{(i)}\right)$, so we may conclude that there are only finitely many critical values of $\|\mu\|^{2}$. Since $\mu$ is proper, there are only finitely many connected components of $\operatorname{Crit}\left(\|\mu\|^{2}\right)$. The final assertion in the theorem follows from [21, Corollary 3.16].

The proof of Theorem 3.1 closely follows the Morse-theoretic argument given in the compact case in $H_{T}^{*}(-; \mathbb{Q})$ in $[28$, Theorem 3], except that we use our $K$-theoretic Atiyah-Bott lemma (Lemma 2.1). Hence we do not give all the details below. Instead, we only briefly indicate why the sum can in fact be restricted to the set $Z$.

Proof of Theorem 3.1. We refer the reader to [28] for details. First, the only point of the proof requiring substantial argument is to prove that $\operatorname{ker}(\kappa) \subseteq \mathcal{K}$. Second, we order the connected components of the critical sets of the norm-square $\|\mu\|^{2}$; denote these as $\left\{C_{i}\right\}_{i=0}^{m}$, where $C_{0}=\mu^{-1}(0)$. Hence $\alpha \in \operatorname{ker}(\kappa)$ exactly means $\left.\alpha\right|_{C_{0}}=0$. Third, by observing that all components of $M^{T}$ are also components of $\operatorname{Crit}\left(\|\mu\|^{2}\right)$, it suffices by Theorem 2.5 to show that there exists a $\beta \in \mathcal{K}$ such that $\left.\alpha\right|_{C_{i}}=\left.\beta\right|_{C_{i}}$ for all $i$. The final and most important step in this argument is an inductive construction of the $\beta$, for which it suffices to show that for $1 \leq \ell \leq m$ and $\alpha \in \operatorname{ker}(\kappa)$ such that $\left.\alpha\right|_{C_{i}}=0$ for all $0 \leq i \leq \ell-1$, there exists an element $\alpha^{\prime} \in \mathcal{K}$ such that $\left.\alpha^{\prime}\right|_{C_{i}}=\left.\alpha\right|_{C_{i}}$ for all $0 \leq i \leq \ell$. The construction given by Tolman and Weitsman can now be explicitly seen to produce an element $\alpha$ which is in fact contained in $\mathcal{K}_{\mu\left(C_{\ell}\right)}$. Since $\mu\left(C_{\ell}\right) \in Z$ by definition (3.1), this implies that $\alpha^{\prime} \in \mathcal{K}$. The rest of the argument follows that in [28].

\section{GKM theory in $K$-theory}

The main result of this section is to show that, under some technical conditions on the orbit stratification, the $K$-theory of a Hamiltonian $T$-space has a combinatorial description, using data from the equivariant one-skeleton. Such a description is useful for explicit computations of the kernel of the Kirwan map. As mentioned in the Introduction, this result is best viewed as part of a large body of work inspired by the original paper of Goresky, Kottwitz, and MacPherson [11]. Both the statement of our theorem and its proof are $K$-theoretic versions of those given in [15, Theorem 2.11], with slight differences in the hypotheses (explained below).

We begin by defining an extra condition on the $T$-action that will be necessary to state the theorem.

Definition 4.1. Let $T$ be a compact torus and $(M, \omega)$ a Hamiltonian $T$-space. We say that the action is GKM if $M^{T}$ consists of finitely many isolated points, and the $T$-isotropy weights at each fixed point $p \in M^{T}$ are pairwise linearly independent in $\mathfrak{t}_{\mathbb{Z}}^{*}$.

Remark 4.2. The GKM condition on the $T$-isotropy weights given in Definition 4.1 is less restrictive than the hypothesis in [15, Theorem 2.11] that the $T$-weights are relatively prime in $H_{T}^{*}(\mathrm{pt} ; \mathbb{Z})$. This is due to the difference between equivariant Euler 
classes in $K_{T}^{*}(\mathrm{pt})$ and those in $H_{T}^{*}(\mathrm{pt} ; \mathbb{Z})$. A more detailed discussion of the differences between the Atiyah-Bott lemma in equivariant $K$-theory and in integral Borel equivariant cohomology can be found in [16, Section 2].

We now briefly recall the construction of the combinatorial and graph-theoretic data used in our theorem. Let $N$ denote the subset of $M$ given by

$$
N:=\{p \in M \mid \operatorname{codim}(\operatorname{Stab}(p))=1\} .
$$

Thus $N$ consists of the points in $M$ whose $T$-orbits are exactly one-dimensional. The equivariant one-skeleton of $M$ is then defined to be the closure $\bar{N}$ of $N$. Hence

$$
\bar{N}=\{p \in M \mid \operatorname{codim}(\operatorname{Stab}(p)) \leq 1\}=N \cup M^{T} .
$$

The GKM condition states that at each fixed point $p \in M^{T}$, the $T$-isotropy weights are pairwise linearly independent in $\mathfrak{t}_{\mathbb{Z}}^{*}$. Each $T$-weight space corresponds to a component of $N$, the closure of which is either an $S^{2} \cong \mathbb{P}^{1}$ (with north and south poles being $T$ fixed points $\{p, q\} \subseteq M^{T}$ ) or a copy of $\mathbb{C}$ (with origin a $T$-fixed point). Thus, given the GKM condition, the one-skeleton $\bar{N}$ is a collection of projective spaces $\mathbb{P}^{1}$ and affine spaces $\mathbb{C}$, glued at $T$-fixed points. By definition, each component of $N$ is equipped with a $T$-action which is specified by a weight in $\mathfrak{t}_{\mathbb{Z}}^{*} \cong \operatorname{Hom}\left(T, S^{1}\right)$; this weight appears in the $T$-weight decomposition of the isotropy action on the corresponding $T$-fixed point. (There is a sign ambiguity in the $T$-weight for a component of $N$ whose closure is a $\mathbb{P}^{1}$, depending on the choice of north or south pole; however, this choice does not affect the GKM computation to be described below.)

From this data we construct the GKM graph, a labelled graph $\Gamma=(V, E, \alpha)$, associated to the GKM $T$-space $M$. The vertices $V$ of $\Gamma$ are the $T$-fixed points $V=M^{T}$, and there is an edge $(p, q) \in E$ exactly when there exists an embedded $\mathbb{P}^{1} \subset \bar{N}$ containing as its two $T$-fixed points $\{p, q\} \subset \mathbb{P}^{1}$. Additionally, we label each edge $(p, q)$ with the weight $\alpha_{(p, q)}$ specifying the $T$-action on the corresponding $\mathbb{P}^{1}$ as discussed above. Note that the components of $N$ corresponding to an affine space $\mathbb{C}$ do not contribute to the GKM graph since each such $\mathbb{C}$ equivariantly retracts to its corresponding $T$-fixed point.

Now we return to the setting of the previous sections. Let $(M, \omega)$ be a symplectic manifold equipped with a Hamiltonian $T$-action. As before, we assume that the moment map $\mu: M \rightarrow \mathfrak{t}^{*}$ has a component which is proper and bounded below. In addition, we now assume that the $T$-action on $M$ is GKM, so in particular $M^{T}$ is a finite set of isolated points in $M$. Let $\mu^{\xi}: M \rightarrow \mathbb{R}$ be a generic component of $\mu$ which is proper and bounded below, as chosen in Lemma 2.3. Then $\mu^{\xi}$ is a Morse function on $M$. Order the critical points $\operatorname{Crit}\left(\mu^{\xi}\right)=M^{T}=\left\{p_{i}\right\}_{i=1}^{m}$ so that $\mu^{\xi}\left(p_{j}\right)<\mu^{\xi}\left(p_{k}\right)$ if and only if $j<k$. Since the Morse function is $T$-invariant, the negative gradient flow with respect to a $T$-invariant metric is $T$-equivariant.

Let $U_{i}$ be the flow-down cell from the critical point $p_{i}$. Then the negative gradient flow gives a $T$-equivariant deformation retraction of $M$ to the union of the $U_{i}$, i.e.,

$$
M \sim \bigcup_{i} U_{i}
$$


is a $T$-equivariant homotopy equivalence. Hence to study the $T$-equivariant $K$-theory of $M$, we may instead study that of $M^{\prime}:=\bigcup_{i} U_{i}$. Each $U_{i}$ contains a single critical point $p_{i}$, and $T_{p_{i}} U_{i}$ is a $T$-representation; by the GKM assumption, $T$-weights occurring in $T_{p_{i}} U_{i}$ are pairwise linearly independent.

We may now use the results of [14] to show that, under the conditions outlined above, the GKM graph $\Gamma$ combinatorially encodes the equivariant $K$-theory of the Hamiltonian $T$-space $M$. We first state a crucial lemma which involves $K$-theoretic equivariant Euler classes in $K_{T}^{*}(\mathrm{pt}) \cong R(T)$, where $R(T)$ is the representation ring of $T$; this will be the key step in the proof of the main theorem. Let $\mathbb{C}_{\sigma}$ be a 1dimensional representation of $T$ with weight $\sigma \in \mathfrak{t}_{\mathbb{Z}}^{*}$. Recall that the $K$-theoretic $T$-equivariant Euler class of the $T$-bundle $\mathbb{C}_{\sigma} \rightarrow \mathrm{pt}$ is

$$
e_{T}(\sigma):=1-e^{-\sigma} \in K_{T}^{*}(\mathrm{pt}) \cong R(T) .
$$

By properties of the Euler class, if $E=\bigoplus_{i} \mathbb{C}_{\sigma_{i}}$ is a direct sum of such 1-dimensional representations, then the equivariant Euler class of $E$ is the product $e_{T}(E)=\prod_{i}(1-$ $\left.e^{-\sigma_{i}}\right)$. The following is a special case of [29, Lemma 4.9]:

Lemma 4.3. Let $\sigma, \tau \in \mathfrak{t}_{\mathbb{Z}}^{*}$ be linearly independent in $\mathfrak{t}_{\mathbb{Z}}^{*}$. Then the corresponding Euler classes $e_{T}(\sigma)=1-e^{-i \sigma}$ and $e_{T}(\tau)=1-e^{-i \tau} \cong R(T)$ are relatively prime in $K_{T}^{*}(\mathrm{pt})=R(T)$.

We will use this lemma to obtain the following combinatorial description of $K_{T}^{*}(M)$. Since $M^{T}$ consists of finitely many isolated points,

$$
K_{T}^{*}\left(M^{T}\right) \cong \bigoplus_{p \in M^{T}} K_{T}^{*}(p) \cong\left\{h: V=M^{T} \rightarrow K_{T}^{*}(\mathrm{pt}) \cong R(T)\right\} .
$$

We then define the $\Gamma$-subring of $K_{T}^{*}\left(M^{T}\right)$ to be

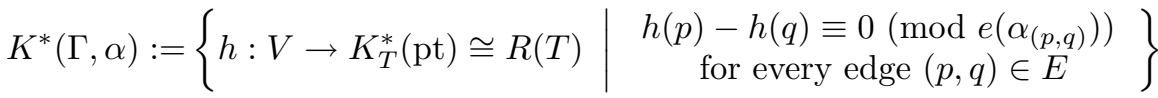

$$
\begin{aligned}
& \subseteq K_{T}^{*}\left(M^{T}\right) \text {. }
\end{aligned}
$$

We have the following:

Theorem 4.4. Let $T$ be a compact torus and $(M, \omega)$ a Hamiltonian $T$-space with moment map $\mu: M \rightarrow \mathfrak{t}^{*}$. Suppose there exists a component of the moment map which is proper and bounded below, and that the T-action on $M$ is GKM. Then the inclusion $\imath: M^{T} \hookrightarrow M$ induces an isomorphism

$$
\imath^{*}: K_{T}^{*}(M) \rightarrow K^{*}(\Gamma, \alpha) \subseteq K_{T}^{*}\left(M^{T}\right) .
$$

Proof. We will use a special case of [14, Theorem 3.1], which states that for $T$-spaces satisfying certain assumptions, the equivariant $K_{T}^{*}$-theory is isomorphic via $\imath^{*}$ to the $\Gamma$-subring defined above. We must therefore check that each of the hypotheses necessary for this theorem is satisfied.

We have already seen that it suffices to compute the $K_{T}^{*}$-theory of the subspace $M^{\prime}:=\bigcup_{i} U_{i}$ of $M$. Let $M_{i}:=\bigcup_{1 \leq j \leq i} U_{i}$ be the union of these cells up to the $i$-th cell. Then $M=\bigcup_{i} M_{i}$ is a $T$-invariant stratification of $M^{\prime}$, and by construction each quotient $M_{i} / M_{i-1}$ is homeomorphic to the Thom space of the $T$-equivariant negative normal bundle $\nu\left(p_{i}\right) \rightarrow p_{i}$ to the fixed point $p_{i}$ with respect to $\mu^{\xi}$. 
Each negative normal bundle $\nu\left(p_{i}\right)$ is $T$-orientable, since it is complex. Moreover, $\nu\left(p_{i}\right)$ decomposes into a direct sum of one-dimensional $T$-weight spaces $\mathbb{C}_{\alpha_{j, p_{i}}}$. Identify the negative normal bundle with $U_{i}=M_{i} \backslash M_{i-1}$. Each $\mathbb{C}_{\alpha_{j, p_{i}}}$ corresponds to a component of $N$, so in particular there is an attaching map of the sphere bundle $S\left(\nu\left(p_{i}\right)\right) \rightarrow M_{i-1}$ which, when restricted to $S\left(\mathbb{C}_{\alpha_{j, p_{i}}}\right)$, maps it to a critical point $p_{j}$, where $j<i$. Such attaching maps exactly correspond to the presence of the embedded $\mathbb{P}^{1} \mathrm{~s}$ in the equivariant one-skeleton.

Moreover, since the $T$-isotropy weights $\alpha_{j, p_{i}}$ are all non-zero, their equivariant Euler classes $1-e^{-\alpha_{j, p_{i}}}$ in $K_{T}^{*}(\mathrm{pt}) \cong R(T)$ are not zero divisors in $K_{T}^{*}(\mathrm{pt})$. Furthermore, by the GKM assumption, the weights at a given critical point $p_{i}$ are also pairwise linearly independent. By Lemma 4.3, this implies that their equivariant Euler classes are pairwise relatively prime in $K_{T}^{*}(\mathrm{pt})$.

By $\left[14\right.$, Theorem 3.1], we may conclude that $K_{T}^{*}(M) \cong K_{T}^{*}\left(M^{\prime}\right)$ injects via $\imath^{*}$ into $K_{T}^{*}\left(M^{T}\right)$ with image exactly the $\Gamma$-subring. This concludes the proof.

\section{Example: symplectic toric manifolds}

In this section, we illustrate the kernel computation procedure outlined in Section 3 by giving an explicit computation of the ordinary $K$-theory of compact symplectic toric manifolds. We thus obtain a description analogous to the Stanley-Reisner presentation of the (equivariant) cohomology ring of toric varieties (see e.g. [29], [5]). The Delzant construction [7] is an explicit construction of a compact symplectic toric manifold, combinatorially specified by a compact Delzant polytope $\Delta \subseteq \mathbb{R}^{n}$, as a symplectic quotient of an affine space $\mathbb{C}^{N}$ by a linear (and hence Hamiltonian) $T^{k}$-action; this allows us to use Theorem 3.1. We only briefly recall the basic ingredients of this Delzant construction below and refer the reader to [6] for details.

Let $\Delta \subseteq \mathbb{R}^{n}$ be a compact Delzant polytope, i.e., it is compact, simple, rational, and smooth. Let $a_{i}$ be the primitive outward-pointing normal vectors to the facets $\left\{F_{i}\right\}_{i=1}^{N}$ of $\Delta$. Hence

$$
\Delta=\left\{x \in \mathbb{R}^{n} \mid\left\langle x, a_{i}\right\rangle \leq \eta_{i}, 1 \leq i \leq N\right\},
$$

for some $\eta_{i} \in \mathbb{R}$. Here $\langle$,$\rangle denotes the standard inner product on \mathbb{R}^{n}$. (As in Section 3 , we identify $\mathbb{R}^{n}$ with its dual using the inner product.) Consider the torus $T^{N}$ acting standardly on $\mathbb{C}^{N}$. The Lie algebra $\mathfrak{t}^{N} \cong \mathbb{R}^{N}$ has standard basis $\left\{\varepsilon_{i}\right\}_{i=1}^{N}$ and its dual $\left(\mathfrak{t}^{N}\right)^{*}$ has dual basis $\left\{u_{i}\right\}_{i=1}^{N}$. Let $\beta: \mathfrak{t}^{N} \rightarrow \mathfrak{t}^{n} \cong \mathbb{R}^{n}$ be the linear map defined by $\beta\left(\varepsilon_{i}\right)=a_{i}$. This gives us an exact sequence of Lie algebras

$$
0 \longrightarrow \mathfrak{t}^{k} \stackrel{\iota}{\longrightarrow} \mathfrak{t}^{N} \stackrel{\beta}{\longrightarrow} \mathfrak{t}^{n} \longrightarrow 0
$$

where $\iota$ is the inclusion of $\mathfrak{t}^{k}:=\operatorname{ker}(\beta)$ into $\mathfrak{t}^{N}$, with $k=N-n$. There is a corresponding exact sequence of Lie groups

$$
1 \rightarrow T^{k} \rightarrow T^{N} \rightarrow T^{n} \rightarrow 1
$$

Hence $\mathbb{C}^{N}$ is also a Hamiltonian $T^{k}$-space, given by restricting the action to the subgroup $T^{k}$. A moment map for this $T^{k}$-action is given by

$$
\Phi\left(z_{1}, \ldots, z_{n}\right)=-\left(\frac{1}{2} \sum_{i=1}^{N}\left|z_{i}\right|^{2} \iota^{*} u_{i}\right)+\iota^{*} \eta
$$


where $\eta=\left(\eta_{1}, \ldots, \eta_{N}\right) \in \mathbb{R}^{N} \cong\left(\mathfrak{t}^{N}\right)^{*}$ is constructed from the constants $\eta_{i}$ in (5.1). The group $T^{k}$ acts freely on $\Phi^{-1}(0)$ and the toric manifold corresponding to $\Delta$ is obtained as the symplectic quotient $X:=\mathbb{C}^{N} / / T^{k}=\Phi^{-1}(0) / T^{k}$. From the construction it may be explicitly seen that $\Phi$ has a component which is proper and bounded below, and the $T^{k}$-action on $\mathbb{C}^{N}$ has only finitely many fixed point components. Hence we may apply Theorem 3.1 to this setting.

Before proceeding to the computation of the kernel, we make a few observations. First, there is a residual torus $T^{n}$ acting on $X$, with moment map image $\Delta$. The facet $F_{i}$ of $\Delta$ is exactly the $T^{n}$-moment map image of $\left(\left\{z_{i}=0\right\} \cap \Phi^{-1}(0)\right) / T^{k}$. In particular, for a subset $A \subseteq\{1,2, \ldots, N\}$,

$$
\bigcap_{i \in A^{c}} F_{i}=\varnothing \quad \Longleftrightarrow \quad \mathbb{C}^{A} \cap \Phi^{-1}(0)=\varnothing,
$$

where $\mathbb{C}^{A} \subseteq \mathbb{C}^{N}$ denotes the coordinate subspace obtained by setting $z_{j}=0$ for $j \in A^{c}$. Second, we observe that $K_{T^{k}}^{*}\left(\mathbb{C}^{N}\right) \cong R\left(T^{k}\right)$ may be expressed as the quotient of

$$
K_{T^{N}}^{*}\left(\mathbb{C}^{N}\right) \cong R\left(T^{N}\right) \cong \mathbb{Z}\left[x_{1}, \ldots, x_{N}, x_{1}^{-1}, \ldots, x_{N}^{-1}\right]
$$

by the ideal

$$
\mathcal{J}=\left\langle x^{\alpha}-1 \mid \alpha \in \beta^{*}\left(\left(\mathfrak{t}^{n}\right)_{\mathbb{Z}}^{*}\right) \subseteq\left(\mathfrak{t}^{N}\right)_{\mathbb{Z}}^{*} \cong \mathbb{Z}^{N}\right\rangle,
$$

where $\left(\mathfrak{t}^{N}\right)_{\mathbb{Z}}^{*} \cong \mathbb{Z}^{N}$ and we write $x^{\alpha}:=x_{1}^{\alpha_{1}} x_{2}^{\alpha_{2}} \cdots x_{N}^{\alpha_{N}}$ for $x=\left(x_{1}, \ldots, x_{N}\right)$, and $\alpha=\left(\alpha_{1}, \ldots, \alpha_{N}\right)$.

We now proceed with the kernel computation. We first prove a technical lemma which allows us to deal with the slight difficulty that an arbitrary component of $\Phi$ need not be proper and bounded below.

Lemma 5.1. Let $T^{k}$ be a compact torus acting linearly on $\mathbb{C}^{N}$ as specified by (5.2), with moment map $\Phi: \mathbb{C}^{N} \rightarrow\left(\mathfrak{t}^{k}\right)^{*}$. Let $\Phi^{\xi}$ be a component of the moment map and $c$ a critical value of $\Phi^{\xi}$. Let $\Sigma_{c}$ be the corresponding critical set of $\Phi^{\xi}$ and let $D\left(\nu_{c}\right), S\left(\nu_{c}\right)$ denote the disc and sphere bundles, respectively, of the negative normal bundle $\nu_{c}$ to $\Sigma_{c}$ with respect to $\Phi^{\xi}$. Let $M_{c}^{+}:=\left(\Phi^{\xi}\right)^{-1}(-\infty, c+\varepsilon)$ and $M_{c}^{-}:=\left(\Phi^{\xi}\right)^{-1}(-\infty, c-\varepsilon)$. Then the negative gradient flow corresponding to $\Phi^{\xi}$ with respect to the standard Kähler metric on $\mathbb{C}^{N}$ gives a $T^{k}$-equivariant retraction of the pair $\left(M_{c}^{+}, M_{c}^{-}\right)$onto $\left(D\left(\nu_{c}\right), S\left(\nu_{c}\right)\right)$.

Proof. Let $F_{\Sigma}$ denote the union of the flow-up and flow-down sets contained in $M_{c}^{+}$ associated to $\Sigma_{c}$. Since an arbitrary component $\Phi^{\xi}$ of the moment map may not be proper or bounded below on the noncompact space $\mathbb{C}^{N}$, we must show that any point $z \in M_{c}^{+} \backslash F_{\Sigma}$ flows down to $M_{c}^{-}$with respect to the negative gradient flow of $\Phi^{\xi}$. From this, the lemma follows.

In our case, the vector field $-\operatorname{grad}\left(\Phi^{\xi}\right)$ is complete by [1, Proposition 2.1.21] so the flow is defined for all $t \in \mathbb{R}$. Suppose that $z \in M_{C}^{+} \backslash F_{\Sigma}$ and let $\gamma(t)$ for $t \in \mathbb{R}$ denote the negative gradient flow through $z$ with $\gamma(0)=z$. It will suffice to show that

$$
\left|d \Phi_{\gamma(t)}^{\xi}\left(\gamma^{\prime}(t)\right)\right|=\left|d \Phi_{\gamma(t)}^{\xi}\left(\operatorname{grad} \Phi^{\xi}\right)_{\gamma(t)}\right|=|| \operatorname{grad}\left(\Phi^{\xi}\right)_{\gamma(t)} \|^{2}
$$


remains bounded away from 0 as $t \rightarrow \infty$. On the other hand, in the case of a linear action of $T^{k}$ on $\mathbb{C}^{N}$, we have

$$
\left\|\operatorname{grad}\left(\Phi^{\xi}\right)_{\gamma(t)}\right\|^{2}=\left\|J \xi_{\gamma(t)}^{\sharp}\right\|^{2}=\left\|\xi_{\gamma(t)}^{\sharp}\right\|^{2}=\|\iota(\xi) \cdot \gamma(t)\|^{2},
$$

where $J$ is the standard complex structure on $\mathbb{C}^{N}$.

Given a $\xi \in \mathfrak{t}^{k}$, let $\iota(\xi)=\left(\xi_{1}, \xi_{2}, \ldots, \xi_{N}\right) \in \mathfrak{t}^{N} \cong \mathbb{R}^{N}$ and let $A \subseteq\{1,2, \ldots, N\}$ be the subset $A=\left\{j: \xi_{j} \neq 0\right\}$. From the formula $\operatorname{grad}\left(\Phi^{\xi}\right)_{y}=J_{\iota}(\xi) y$, for any $y \in \mathbb{C}^{N}$, we see that for $\ell \in A^{c}$, the coordinate $\gamma(t)_{\ell}=\gamma(0)_{\ell}=z_{\ell}$ is constant for all $t \in \mathbb{R}$. Now let $C>0$ be such that $\left|\iota(\xi)_{j}\right|^{2}>C$ for all $j \in A$. Let $\pi_{\xi}: \mathbb{C}^{N} \rightarrow \mathbb{C}^{A}$ be the linear projection which sends to 0 all coordinates $z_{\ell}$ for $\ell \in A^{c}$. Then we have the estimate

$$
\left\|\operatorname{grad}\left(\Phi^{\xi}\right)_{\gamma(t)}\right\|^{2}>C\left\|\pi_{\xi}(\gamma(t))\right\|^{2}
$$

for all $t>0$. Suppose now that $\left\|\operatorname{grad}\left(\Phi^{\xi}\right)_{\gamma(t)}\right\|^{2} \rightarrow 0$ as $t \rightarrow \infty$. Then $\left\|\pi_{\xi}(\gamma(t))\right\|^{2} \rightarrow 0$ also, and in particular $\gamma(t)$ remains in a compact set. Hence $\gamma(t)$ converges. For a linear action of $T^{k}$ on $\mathbb{C}^{N}, \Phi^{\xi}$ has only one critical component $\Sigma_{c}$, so $\gamma(t)$ must converge to $\Sigma_{c}$, and we conclude $z \in F_{\Sigma}$. Therefore, if $z \in M_{C}^{+} \backslash F_{\Sigma}$, then $\left\|\operatorname{grad}\left(\Phi^{\xi}\right)_{\gamma(t)}\right\|^{2}$ is bounded away from 0 for all $t$ and $z$ flows down to $M_{c}^{-}$.

With this lemma in hand, we may prove our main theorem.

Theorem 5.2. Let $\Delta \subseteq \mathbb{R}^{n}$ be a compact Delzant polytope with facets $\left\{F_{i}\right\}_{i=1}^{N}$ as specified in (5.1). Let $X:=\mathbb{C}^{N} / / T^{k}$ be the symplectic quotient of $\mathbb{C}^{N}$ corresponding to $\Delta$, as above. Then the ordinary $K$-theory ring $K^{*}(X)$ is given by

$$
K^{*}(X) \cong \mathbb{Z}\left[x_{1}, \ldots, x_{N}, x_{1}^{-1}, \ldots, x_{N}^{-1}\right] / \mathcal{I}+\mathcal{J},
$$

where

$$
\mathcal{I}=\left\langle\prod_{i \in S}\left(1-x_{i}^{-1}\right) \mid \bigcap_{i \in S} F_{i}=\varnothing\right\rangle
$$

and

$$
\mathcal{J}=\left\langle x^{\alpha}-1 \mid \alpha \in \beta^{*}\left(\left(\mathfrak{t}^{n}\right)_{\mathbb{Z}}^{*}\right) \subseteq\left(\mathfrak{t}^{N}\right)_{\mathbb{Z}}^{*} \cong \mathbb{Z}^{N}\right\rangle .
$$

Proof. We have already observed that $K_{T^{N}}^{*}\left(\mathbb{C}^{N}\right) / \mathcal{J}$ is isomorphic to $K_{T^{k}}^{*}\left(\mathbb{C}^{N}\right)$, so to prove the theorem it suffices to describe $\operatorname{ker}(\kappa)$ in terms of the generators in $K_{T^{N}}^{*}\left(\mathbb{C}^{N}\right) \cong K_{T^{N}}^{*}(\mathrm{pt})$. By Theorem 3.1, we must compute $K_{\xi}$ for each $\xi \in Z$ as in $(3.1)$.

Since $T^{k}$ is acting linearly on $\mathbb{C}^{N}$, for each subset $A \subseteq\{1,2, \ldots, N\}$ there is a unique critical value $\xi_{A} \in \Phi\left(\mathbb{C}^{A}\right)=\operatorname{span}_{\mathbb{R}_{+}}\left\{\alpha_{i}\right\}_{i \in A}-\eta$ associated to the linear subspace $\mathbb{C}^{A}$, and $Z$ consists of the set of all $\xi_{A}$. (Here we denote by $\operatorname{span}_{\mathbb{R}_{+}}\left\{\alpha_{i}\right\}_{i \in A}$ the set of linear combinations of $\alpha_{i}$ with non-negative coefficients.) We note first that since 0 is the only $T^{k}$-fixed point of $\mathbb{C}^{N}$, and any class which restricts to 0 at the critical point 0 is trivial, it suffices to consider $\xi_{A}$ such that $\left\langle\eta, \xi_{A}\right\rangle<0$. It also suffices to consider only $\xi_{A}$ such that $\eta \notin \operatorname{span}_{\mathbb{R}_{+}}\left\{\alpha_{i}\right\}_{i \in A}$, which is equivalent to $\bigcap_{j \in A^{c}} F_{j}=\varnothing$ by (5.3), since otherwise $\xi_{A}=0$ and $K_{\xi_{A}}=\{0\}$.

We now show that for every $\xi_{A} \in Z$ with $\bigcap_{j \in A^{c}} F_{j}=\varnothing$, the ideal $K_{\xi_{A}}$ is generated by an element of the form $\prod_{i \in S}\left(1-x_{i}^{-1}\right)$ for $S$ such that $\bigcap_{i \in S} F_{i}=\varnothing$. By definition of $Z$, the element $\xi_{A}$ has the shortest norm in $\operatorname{span}_{\mathbb{R}_{+}}\left\{\alpha_{i}\right\}_{i \in A}$, so by construction 
$\xi_{A}$ has the property $\left\langle\alpha_{i}, \xi_{A}\right\rangle=0$ for all $i \in A$. Moreover, $\Phi$ is proper, so we have $\left\langle\eta, \xi_{A}\right\rangle<0$. By Theorem 2.5, in order to determine $K_{\xi_{A}}$ it suffices to examine the behavior of a class in $K_{\xi_{A}}$ at the unique $T^{k}$-fixed point $0 \in \mathbb{C}^{N}$. Let

$$
M_{0}^{+}:=\left(\Phi^{\xi_{A}}\right)^{-1}(-\infty,+\varepsilon), \quad M_{0}^{-}:=\left(\Phi^{\xi_{A}}\right)^{-1}(-\infty,-\varepsilon)
$$

for $0<\varepsilon<\left\langle-\eta, \xi_{A}\right\rangle$. By Lemma 5.1 we know that the pair $\left(M_{0}^{+}, M_{0}^{-}\right)$is $T^{k}$ equivariantly homotopic to the disc and sphere bundle pair $\left(D\left(\nu_{0}\right), S\left(\nu_{0}\right)\right)$. The negative normal bundle to the critical set of $\Phi^{\xi_{A}}$ is spanned by the coordinate directions $j$ such that $\left\langle\alpha_{j}, \xi_{A}\right\rangle<0$. Let $S$ be the subset of such $j$. Then by construction we have $\eta \notin \operatorname{span}_{\mathbb{R}_{+}}\left\{\alpha_{i}\right\}_{i \in S^{c}}$ and thus $\bigcap_{i \in S} F_{i}=\varnothing$. Lemma 5.1 and our choice of $\varepsilon$ also imply that $M_{0}^{-}$is $T^{k}$-equivariantly homotopic to $M_{\xi_{A}}$. These observations in addition to an analysis of the long exact sequence of the pair $\left(M_{0}^{+}, M_{0}^{-}\right)$imply that $K_{\xi_{A}}$ is generated by the class in

represented by $\prod_{i \in S}\left(1-x_{i}^{-1}\right)$.

$$
K_{T^{n}}^{*}\left(\mathbb{C}^{N}\right) \cong K_{T^{N}}^{*}\left(\mathbb{C}^{N}\right) / \mathcal{J} \cong R\left(T^{N}\right) / \mathcal{J}
$$

Finally, we show that for every $S$ such that $\bigcap_{i \in S} F_{i}=\varnothing$, there exists $A$ such that that the product $\prod_{i \in S}\left(1-x_{i}^{-1}\right)$ is contained in $K_{\xi_{A}}$. In fact it suffices to show this for minimal such $S$. By definition, for $A:=S^{c}$ we have that $\eta \notin \operatorname{span}_{\mathbb{R}_{+}}\left\{\alpha_{i}\right\}_{i \in A}$. Hence $\xi_{A}$ is non-zero and $K_{\xi_{A}}$ is nontrivial. Moreover, since $S$ is minimal, we have $\left\langle\xi_{A}, \alpha_{i}\right\rangle<0$ for all $i \in S$. Hence each coordinate line for $i \in S$ is contained in the negative normal bundle to the critical set $\mathbb{C}^{S}$ for the moment map component $\Phi^{\xi_{A}}$, and so by the argument above $K_{\xi_{A}}$ contains the product $\prod_{i \in S}\left(1-x_{i}^{-1}\right)$. This completes the proof.

\section{Acknowledgements}

We thank Jonathan Weitsman for helpful discussions. The second author thanks the University of Toronto and the Fields Institute for their hospitality and support while conducting a portion of this research. Both authors thank the American Institute of Mathematics and the Banff International Research Station for their hospitality.

\section{References}

[1] R. Abraham and J. E. Marsden, Foundations of mechanics. Benjamin/Cummings Publishing Co. Inc. Advanced Book Program, Reading, Mass., 1978. Second edition, revised and enlarged.

[2] M. F. Atiyah, Bott periodicity and the index of elliptic operators, Quart. J. Math. Oxford Ser. (2), 19 (1968) 113-140.

[3] M. F. Atiyah and R. Bott, The Yang-Mills equations over Riemann surfaces, Philos. Trans. Roy. Soc. London Ser. A, 308 (1983), no. 1505, 523-615.

[4] M. F. Atiyah and G. Segal, Twisted K-theory, Ukr. Mat. Visn. 1 (2004), no. 3, 287-330.

[5] L. A. Borisov and R. P. Horja, On the K-theory of smooth toric DM stacks, In Snowbird lectures on string geometry, 21-42, Contemp. Math., 401, Amer. Math. Soc., Providence, RI, 2006.

[6] A. Cannas da Silva, Lectures on symplectic geometry, volume 1764 of Lecture Notes in Mathematics. Springer-Verlag, Berlin, 2001.

[7] T. Delzant, Hamiltoniens périodiques et images convexes de l'application moment, Bull. Soc. Math. France, 116 (1988), no. 3, 315-339.

[8] R. Goldin, T. S. Holm, and A. Knutson, Orbifold cohomology of torus quotients, Duke Math. J. 139 (2007), no. 1, 89-129.

[9] R. F. Goldin, The cohomology rings of weight varieties and polygon spaces, Adv. Math. 160 (2001), no. 2, 175-204. 
[10] An effective algorithm for the cohomology ring of symplectic reductions, Geom. Funct. Anal. 12 (2002), no. 3, 567-583.

[11] M. Goresky, R. Kottwitz, and R. MacPherson, Equivariant cohomology, Koszul duality, and the localization theorem, Invent. Math. 131 (1998), 25-83.

[12] V. Guillemin, V. Ginzburg, and Y. Karshon, Moment maps, cobordisms, and Hamiltonian group actions, volume 98 of Mathematical Surveys and Monographs. American Mathematical Society, Providence, RI, 2002.

[13] V. Guillemin and M. Kogan, Morse theory on Hamiltonian $G$-spaces and equivariant K-theory, J. Differential Geom. 66 (2004), no. 3, 345-375.

[14] M. Harada, A. Henriques, and T. S. Holm, Computation of generalized equivariant cohomologies of Kac-Moody flag varieties, Adv. Math. 197 (2005), no. 1, 198-221.

[15] M. Harada and T. S. Holm, The equivariant cohomology of hypertoric varieties and their real loci, Comm. Anal. Geom. 13 (2005), no. 3, 527-559.

[16] M. Harada and G. D. Landweber, Surjectivity for Hamiltonian G-spaces in K-theory, Trans. Amer. Math. Soc. 259 (2007), no. 12, 6001-6025.

[17] T. Hausel and N. Proudfoot, Abelianization for hyperkähler quotients, Topology, 44 (2005), no. $1,231-248$.

[18] J. C. Hausmann and A. Knutson, Polygon spaces and Grassmannians, L'enseignement Mathématique, 43 (1997), 173-198.

[19] _ The cohomology rings of polygon spaces Ann. Inst. Fourier, Grenoble, 48 (1998), no. $1,281-321$.

[20] T. J. Jarvis, R. Kaufmann, and T. Kimura, Stringy K-theory and the Chern character, Invent. Math. 168 (2007), no. 1, 23-81.

[21] F. Kirwan, Cohomology of quotients in symplectic and algebraic geometry, volume 31 of Mathematical Notes, Princeton University Press, Princeton, N.J., 1984.

[22] _ Momentum maps and reduction in algebraic geometry, Differential Geom. Appl. 9 (1998) no. 1-2, 135-171.

[23] A. Knutson and I. Rosu, Appendix to Equivariant K-theory and equivariant cohomology, Math. Z. 243 (2003), 423-448.

[24] H. Konno, On the cohomology ring of the hyperkähler analogue of the polygon spaces, In Integrable systems, topology, and physics (Tokyo, 2000), 129-142, Contemp. Math. 309, Amer. Math. Soc., Providence, RI, 2002.

[25] E. Lerman, E. Meinrenken, S. Tolman, and C. Woodward, Non-abelian convexity by symplectic cuts, Topology, 37 (1998), no. 2, 245-259.

[26] G. Segal, Equivariant K-theory, Inst. Hautes Études Sci. Publ. Math. 34 (1968), 129-151.

[27] S. Tolman and J. Weitsman, On the cohomology rings of Hamiltonian T-spaces, Proc. of the Northern California Symplectic Geometry Seminar, AMS Translations Series 2, 196 (1999), $251-258$.

[28] $751-773$.

[29] G. Vezzosi and A. Vistoli, Higher algebraic K-theory for actions of diagonalizable groups, Invent. Math. 153 (2003), no. 1, 1-44.

Department of Mathematics and Statistics, McMaster University, 1280 Main Street

West, Hamilton, Ontario L8S 4K1, Canada

E-mail address: Megumi.Harada@math.mcmaster.ca

URL: http://www.math.mcmaster.ca/Megumi.Harada/

Department of Mathematics, Bard College, P.O. Box 5000, Annandale-on-Hudson, Ny 12504-5000, U.S.A.

E-mail address: gregland@bard.edu 